\title{
Anti-SARS-CoV-2 Antibody Screening in Healthcare Workers and Its Correlation with Clinical Presentation in Tertiary Care Hospital, Kathmandu, Nepal, from November 2020 to January 2021
}

\author{
Suraj Aryal $\mathbb{D}$, ${ }^{1}$ Sanskriti Pandit ${ }^{\mathbb{D}},{ }^{1}$ Sushant Pokhrel $\mathbb{D}^{1},{ }^{1}$ Mandira Chhusyabaga ${ }^{(D)}{ }^{1}$ \\ Pabitra Bista $\left(\mathbb{D},{ }^{1}\right.$ Mahendra Psd. Bhatt $\mathbb{D}^{\mathbb{D}},{ }^{1}$ Dharma Datta Subedi $\mathbb{D}^{2},{ }^{2}$ and Basista Psd. Rijal $\mathbb{C}^{3}$ \\ ${ }^{1}$ Department of Laboratory Medicine, Manmohan Memorial Institute of Health Sciences, Kathmandu, Nepal \\ ${ }^{2}$ Department of General Medicine, Manmohan Memorial Medical College and Teaching Hospital, Kathmandu, Nepal \\ ${ }^{3}$ Department of Clinical Microbiology, Manmohan Memorial Medical College and Teaching Hospital, Kathmandu, Nepal \\ Correspondence should be addressed to Basista Psd. Rijal; basistarijal@gmail.com
}

Received 25 June 2021; Revised 28 December 2021; Accepted 6 January 2022; Published 1 February 2022

Academic Editor: Morteza Saki

Copyright (c) 2022 Suraj Aryal et al. This is an open access article distributed under the Creative Commons Attribution License, which permits unrestricted use, distribution, and reproduction in any medium, provided the original work is properly cited.

\begin{abstract}
Background. Antibody titer and the life span of antibodies against SARS-CoV-2 have been found to be associated with the clinical presentation in individuals. The extent of exposure of healthcare workers and the general public to SARS-CoV-2 needs to be assessed to monitor the COVID-19 pandemic. Thus, this study is an attempt in assessing the anti-SARS-CoV-2 antibody in health care workers. Methods. This laboratory-based cross-sectional study was performed in Manmohan Memorial Medical College and Teaching Hospital, Kathmandu from November 2020 to January 2021. A total of 185 HCWs were enrolled in this study. Their serum samples were screened for anti-SARS-CoV-2 antibodies, and a structured questionnaire was administered to collect further information. Anti-SARS-CoV-2 antibody screening was performed using lateral flow immunoassay. The data were analyzed using SPSS version 20. Results. Among $185 \mathrm{HCWs}$ that participated in the study, 41 (22.2\%) tested positive for the anti-SARS-CoV-2 antibody. Of these $41 \mathrm{HCWs}, 37$ tested positive for IgG only and 4 of them tested positive for both IgM and IgG antibodies. The presence of the previous history of SARS-CoV-2 infection $(p<0.001)$, the presence of flu-like symptoms within the last 6 months $(p<0.001)$, and the presence of positive contact history $(p=0.002)$ were statistically significant with the presence of the antibody among HCWs. Conclusion. Healthcare workers carry a high burden of SARS-CoV-2 infection and are at risk of acquiring infection from their workplace. Anti-SARS-CoV-2 antibody screening among healthcare workers is highly recommended in multiple healthcare settings as it can help in monitoring transmission dynamics and evaluation of infection control policies.
\end{abstract}

\section{Introduction}

Severe acute respiratory syndrome coronavirus 2 (SARS$\mathrm{CoV}-2)$ is an enveloped nonsegmented positive sense RNA $\beta$-coronavirus of the Coronaviridae family [1]. It was first diagnosed in December 2019 in Wuhan, China in a patient showing symptoms of atypical pneumonia [2]. The virus was found to cause a new disease called coronavirus disease 2019 (COVID-19) having high infectious potential [3]. Since the first case was identified in China, it has spread globally, and it has been declared a pandemic by the World Health
Organization (WHO) on 11 March 2020. As of 24 June 2021, there have been 179,241,734 confirmed cases of COVID-19 globally, resulting in $3,889,723$ deaths. On the same date, the total number of confirmed cases in Nepal has reached 629,431 , with a total number of 8,918 deaths [4].

Most of the cases of COVID-19 are asymptomatic or with mild symptoms. Individuals characterized as high-risk groups (old aged, diabetic, hypertensive, and cancer patients, individuals with pulmonary diseases or disorders) were found to develop severe clinical presentations leading to multiple organ failure and death [5]. The principle of 
immune response and development of antibodies against SARS-CoV-2 is being studied globally. Infection with SARSCoV-2 evokes the development of IgM and IgG antibodies, which is useful in the assessment of immune response following infection. These antibodies can be detected in the serum within 1 to 3 weeks after the onset of illness [6]. Antibody titer and the lifespan of antibodies against SARSCoV-2 have been found to be determined by the clinical presentation as well. Short-lived and low antibody titers near the detection limit were found in the cases of mild infections [7].

Healthcare workers (HCWs) are considered a high-risk group for SARS-CoV-2 infection. They may acquire infection either from the healthcare settings or from the community. Several studies on the prevalence of SARS-CoV-2 antibodies among HCWs have reported the seroprevalence ranging from $1.6 \%$ to $45.3 \%$ [8]. According to the study performed by Poulikakos et al. in a tertiary care center in North West England, 6\% of the healthcare workers tested positive for SARS-CoV-2 IgG antibody [9]. The study performed by Steensels et al. in Belgium showed that $6.4 \%$ of healthcare workers tested positive for SARS-CoV-2 IgG antibody [10]. The study performed by Mascola et.al.in New York showed that $13.7 \%$ of healthcare workers were seropositive for anti-SARS-CoV-2 antibodies [11]. Exposure to a large number of patients (either symptomatic or asymptomatic) in the hospital for a longer period may be the most common cause of infection for healthcare workers $[8,12]$. Serological assays could be an important method for screening infections and epidemiological studies [13] and also for detecting symptomatic and asymptomatic infections [8]. Thus, this study aims to assess the seroprevalence of antiSARS-CoV-2 antibodies among healthcare workers and to determine the association between clinical presentations and antibody screening in a tertiary care hospital in Kathmandu, Nepal. Furthermore, this study could be useful in assessing the exposure of HCWs to SARS-CoV-2 that will help to assess and monitor transmission dynamics and in the evaluation of infection control policies that would ultimately help in managing the COVID-19 pandemic. It is also recommended by international health authorities to screen for SARS-CoV-2 infection as it would be useful in reducing morbidity, transmission rate, and maintaining the health system within the balance.

\section{Methods}

A laboratory-based cross-sectional study was performed in Manmohan Memorial Medical College and Teaching Hospital (MMTH), Kathmandu, Nepal, during the period of 3 months (November 2020 to January 2021).

2.1. Selection of Study Population. HCWs, including administrative staff working at MMTH at least 6 months before the sample collection date, were invited to participate in the study voluntarily. The study was conducted in all departments of the hospital. All HCWs providing informed written consent were conveniently selected for the study and were requested to fill out the corresponding questionnaire to collect the required information, and HCWs who refused to give the informed written consent $(n=16)$ were excluded from the study. The professional category of the individuals participating in the study was categorized into three risk groups based on the degree of exposure to COVID-19 as [14] follows:

(1) High-risk group: professionals working in a clinical environment with prolonged direct contact with patients (e.g., nurse, doctor)

(2) Moderate risk group: professionals working in a clinical environment with nonintense/no direct patient contact but are at higher risk of nosocomial exposure (e.g., laboratory professionals, support staff)

(3) Low-risk group: professionals working in a nonclinical environment with no patient contact or minimal patient contact (e.g., administrative staff)

2.2. Experimental Protocol. Blood samples were collected from HCWs after taking informed written consent by venipuncture following standard operating protocol. Samples were collected in a serum separator tube (HEBEI XINLE SCI \& TECH CO.LTD, China) containing gel after obtaining informed and written consent.

Samples were centrifuged to obtain serum. Antibody screening was performed from the serum using SARS-CoV2 IgM/IgG Antibody Assay Kit (Colloidal Gold Method) (Zybio Inc., China) as per the manufacturer's instruction ( $5 \mu \mathrm{l}$ serum was added to the sample well using a pipette, followed by the addition of 2 drops of buffer), the results were read within 10 to 15 minutes, and the results after 15 minutes were considered invalid.

The test was validated by positive and negative controls. The serum from the patient with a history of PCR confirmed SARS-CoV-2 infection within the last 3 weeks was used as a positive control, and the serum of a healthy individual without any contact history and any history of SARS-CoV-2 infection was used as a negative control.

2.3. Statistical Analysis. The data were collected in Microsoft Excel 2013 and analyzed using SPSS version 20.0 (IBM Corp., Armonk, NY, USA). Age was the only continuous variable defined using median and interquartile range (IQR). Mann-Whitney $U$ test was used for assessing group differences in age. Categorical variables were expressed as frequency rates $(N)$ and percentages (\%). The chi-square test or Fisher exact test was used as applicable to test for association between categorical variables. $p$ value $<0.05$ was considered statistically significant.

\section{Results}

A total of $185 \mathrm{HCW}$ s participated in the study, and 41 (22.2\%) of them tested positive for the anti-SARS-CoV-2 antibody. Among these 41 individuals, 37 tested positive for 
IgG antibody, while 4 tested positive for both IgM and IgG antibodies (Figure 1). All 4 individuals who tested positive for both IgM and IgG had a recent history of SARS-CoV-2 infection within a month.

The median age of HCWs was 27 (IQR 24-36) years, and $115(62.1 \%)$ of them were in the age group of 20 years to 30 years. Overall, $52(28.1 \%)$ male and 133 (71.9\%) female HCWs participated in the study. Among the 41 individuals, $15(36.6 \%)$ males and 26 (63.4\%) females tested positive for the antibody.

The maximum number of individuals who tested positive for antibodies were nurses (39.0\%), followed by laboratory professionals (19.5\%), administrative staff (17.1\%), doctors (14.6\%), and supporting staff (9.8\%), respectively (Table 1).

Of the total of 185 participants, 113 were of the high-risk group, and among them, $11.89 \%$ (22/185) had positive antibody screening. Similarly, 47 had moderate risk, and 25 were with low risk. The prevalence of antibody positivity was found to be $6.4 \%(12 / 185)$ in the moderate-risk group and $3.78 \%(7 / 185)$ in the low-risk group, respectively (Table 2 ).

Among the 185 individuals tested, 39 (21.1\%) individuals had a previous history of PCR confirmed SARS-CoV-2 infection, and among those 39 individuals, only 21 of them tested antibody positive, whereas 18 of them tested negative for the antibody. All of the 18 subjects who tested negative for the antibody had an asymptomatic infection, and none of them had a history of severe illness.

Overall, 48 (25.9\%) individuals had a history of flu-like symptoms within the last 6 months, and 27 of them were found to be antibody positive. Among 27 of the symptomatic antibody-positive individuals, body ache, headache, fever, dry cough, and fatigue were predominant symptoms, followed by sore throat, runny nose, anosmia, ageusia, and dyspnea (Figure 2).

All 41 individuals who tested positive for antibodies had positive contact history with the SARS-CoV-2 infected patients. The presence of the previous history of SARS-CoV2 infection $(p<0.001)$, the presence of flu-like symptoms within the last 6 months $(p<0.001)$, and the presence of positive contact history $(p=0.002)$ were statistically significant with antibody presence among HCWs (Table 1).

\section{Discussion}

COVID-19 is a global pandemic, infecting more than 179 million people around the globe, causing death to more than 3.8 million as of $24^{\text {th }}$ June 2021 . It is believed that almost all immune-competent individuals after being infected with SARS-CoV-2 will develop an immune response against it [6]. HCWs are considered a high-risk group for SARS-CoV2 infection. They may acquire infection either from the healthcare settings or from the community. Exposure to a large number of patients (either symptomatic or asymptomatic) in the hospital for a longer period may be the most common cause of infection for healthcare workers $[8,9]$.

The seroprevalence of antibodies against SARS-CoV-2 among HCWs in our study was 22.2\%. The "Enhanced Surveillance on Seroprevalence of SARS-CoV-2 in General
Population" of Nepal performed by the Government of Nepal, Ministry of Health and Population in collaboration with WHO in the second and third week of October 2020 found the seroprevalence in the general population to be $14.4 \%$ [15]. Hence, it showed that seroprevalence among HCWs is higher in comparison to the seroprevalence in the general population of Nepal. Similarly, the study performed by Varona et al. in over $6000 \mathrm{HCW}$ s in Spain found higher seroprevalence in HCWs than in the general population [14].

Our study revealed significantly higher seroprevalence among healthcare workers compared to the previously published reports, ranging prevalence rates from $1.26 \%$ to $19.1 \%$. A study performed by Psichogiou et al. in Greece found the seroprevalence rate to be $1.26 \%$ and mentioned the low burden of COVID-19 in Greece could be the reason for lower seroprevalence in the study [8]. The study performed by von Huth et al. in 7950 HCWs in Denmark found the seroprevalence rate to be $2.1 \%$. [16]. Another study by Varona et al. in over 6000 HCWs in Spain found the seroprevalence rate to be $11.0 \%$ [14]. The study performed by Rudberg et al. in Sweden found the seroprevalence rate to be $19.1 \%$ [17]. A similar study by Lombardi et al. in Italy revealed the Seroprevalence rate to be $7.6 \%$ [18]. The higher seroprevalence in our study corresponds with the $24.4 \%$ prevalence rate as reported by Shields et al. in the UK [19].

Galanis et al., in their study, showed that the overall seroprevalence of anti-SARS-CoV-2 antibodies among HCWs was $8.7 \%$, ranging from $0 \%$ to $45.3 \%$. Higher seroprevalence was found in the studies conducted in North America (12.7\%) as compared to the studies conducted in Europe (8.5\%), Africa (8.2\%), and Asia (4\%) [20]. A similar study performed by Hossain et al. found a higher rate of seroprevalence in the USA (12.4\%) compared to the seroprevalence rates in Europe (7.7\%) and Asia (4.8\%) [21]. The study performed by Houlihan et al. in London reported that $45.3 \%$ of HCWs were seropositive [22], and Vaselli et al. reported that the seroprevalence was higher among the HCWs in the UK compared to the HCWs in the rest of Europe during the months of March and August 2020 [23]. Likewise, the study by Müller et al. in the African countries found the seroprevalence to range from $0 \%$ to $45.1 \%$ [24]. Seroprevalence was higher in the study conducted in Nigeria (45.1\%) compared with the study conducted in Libya $(0 \%)$, Egypt (1.3\%), and Togo (1.4\%) [25-28]. Our study revealed relatively higher seroprevalence among HCWs as compared to the reports. The lack of programs for the regular screening of HCWs for SARS-CoV-2 infection, long-term exposure of HCWs with the infected cases (symptomatic or asymptomatic), and lack of proper PPE might explain the relatively higher seroprevalence in our study [29]. In the study performed by Lahner et al. in Italy, it was discussed that longer working hours, the improper use of personal protective equipment (PPE), and long-term exposure to patients could be the risk factors for HCWs for acquiring the disease [13].

In our study, $39.0 \%$ of seropositive HCWs were nurses, suggesting that the seroprevalence is relatively higher among nursing staff than in other HCWs. It could be because of the direct involvement of nursing staff in patient care and treatment, or it could be because of the higher number of 


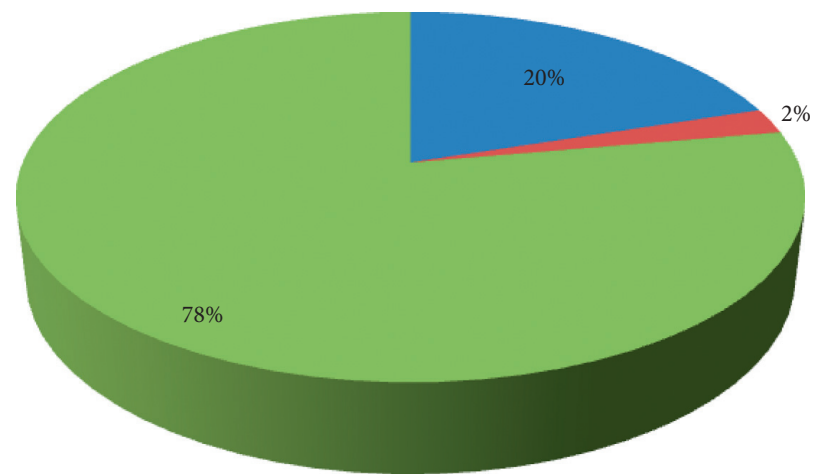

- IgG positive

- IgM and IgG positive

- Antibody negative

Figure 1: Antibody screening among HCWs.

TABLE 1: Subjects characteristics and group differences.

\begin{tabular}{|c|c|c|c|c|}
\hline Characteristics & Antibody positive $N(\%)$ & Antibody negative $N(\%)$ & Total N (\%) & $p$ value \\
\hline Overall & $41(22.2)$ & $144(77.8)$ & 185 & \\
\hline \multicolumn{5}{|l|}{ Age (years) } \\
\hline Median (IQR) & $27(22-35.5)$ & $28(24-36)$ & $27(24-36)$ & 0.766 \\
\hline \multicolumn{5}{|l|}{ Age groups } \\
\hline $20-30$ & $26(63.4)$ & $89(61.8)$ & $115(62.1)$ & \\
\hline $31-40$ & $11(26.9)$ & $35(24.3)$ & $46(24.9)$ & \\
\hline $41-50$ & $3(7.3)$ & $16(11.1)$ & $19(10.3)$ & \\
\hline Above 50 & $1(2.4)$ & $4(2.8)$ & $5(2.7)$ & 0.906 \\
\hline \multicolumn{5}{|l|}{ Gender } \\
\hline Male & $15(36.6)$ & $37(25.7)$ & $52(28.1)$ & \\
\hline Female & $26(63.4)$ & $107(74.3)$ & $133(71.9)$ & 0.171 \\
\hline \multicolumn{5}{|l|}{ Working department } \\
\hline Administration & $7(17.0)$ & $19(13.2)$ & $26(14.1)$ & \\
\hline COVID-19 ward & $3(7.3)$ & $15(10.4)$ & $18(9.7)$ & \\
\hline Emergency & $3(7.3)$ & $13(9.0)$ & $16(8.6)$ & \\
\hline General ward & $5(12.2)$ & $19(13.2)$ & $24(13.0)$ & \\
\hline ICU & $5(12.2)$ & $19(13.2)$ & $24(13.0)$ & \\
\hline Laboratory & $9(22.0)$ & $25(17.4)$ & $34(18.4)$ & \\
\hline OPD & $9(22.0)$ & $34(23.6)$ & $43(23.2)$ & 0.976 \\
\hline \multicolumn{5}{|l|}{ Nature of job } \\
\hline Doctor & $6(14.6)$ & $20(13.9)$ & $26(14.1)$ & \\
\hline Nurse & $16(39.0)$ & $71(49.3)$ & $87(47.0)$ & \\
\hline Laboratory professional & $8(19.5)$ & $20(13.9)$ & $28(15.1)$ & \\
\hline Administrative staff & $7(17.1)$ & $18(12.5)$ & $25(13.5)$ & \\
\hline Support staff & $4(9.8)$ & $15(10.4)$ & $19(10.3)$ & 0.753 \\
\hline \multicolumn{5}{|c|}{ Previous COVID-19 history with positive RT-PCR } \\
\hline Yes & $21(51.2)$ & $18(12.5)$ & $39(21.1)$ & \\
\hline No & $20(48.8)$ & $126(87.5)$ & $146(78.9)$ & $<0.001$ \\
\hline \multicolumn{5}{|c|}{ Presence of flu-like symptoms within 6 months } \\
\hline Yes & $27(65.9)$ & $21(14.6)$ & $48(25.9)$ & \\
\hline No & $14(34.1)$ & $123(85.4)$ & $137(74.1)$ & $<0.001$ \\
\hline \multicolumn{5}{|l|}{ Contact history } \\
\hline Yes & $41(100)$ & $118(81.9)$ & $159(85.9)$ & \\
\hline No & $0(0)$ & $26(18.1)$ & $26(14.1)$ & 0.002 \\
\hline \multicolumn{5}{|l|}{ Underlying illness } \\
\hline Yes & $3(7.3)$ & $7(4.9)$ & $10(5.4)$ & \\
\hline No & $38(92.7)$ & $137(95.1)$ & $175(94.6)$ & 0.695 \\
\hline
\end{tabular}

nursing staff participating in this study in comparison to other HCWs. Similar to our study, Wilkins et al. in Chicago reported nurses as the highest risk group for obtaining infection [30], and a study performed by Al Maskari et al. in Oman reported that $38 \%$ of the infected HCWs were nurses [12]. 
TABLE 2: Risk group based on the degree of exposure to COVID-19.

\begin{tabular}{lccc}
\hline & & Antibody screening & \\
& Positive & Negative & Total $(N)$ \\
\hline High risk & 22 & 91 & 113 \\
Moderate risk & 12 & 35 & 47 \\
Low risk & 7 & 18 & 25 \\
Total $(N)$ & 41 & 144 & 185 \\
\hline
\end{tabular}

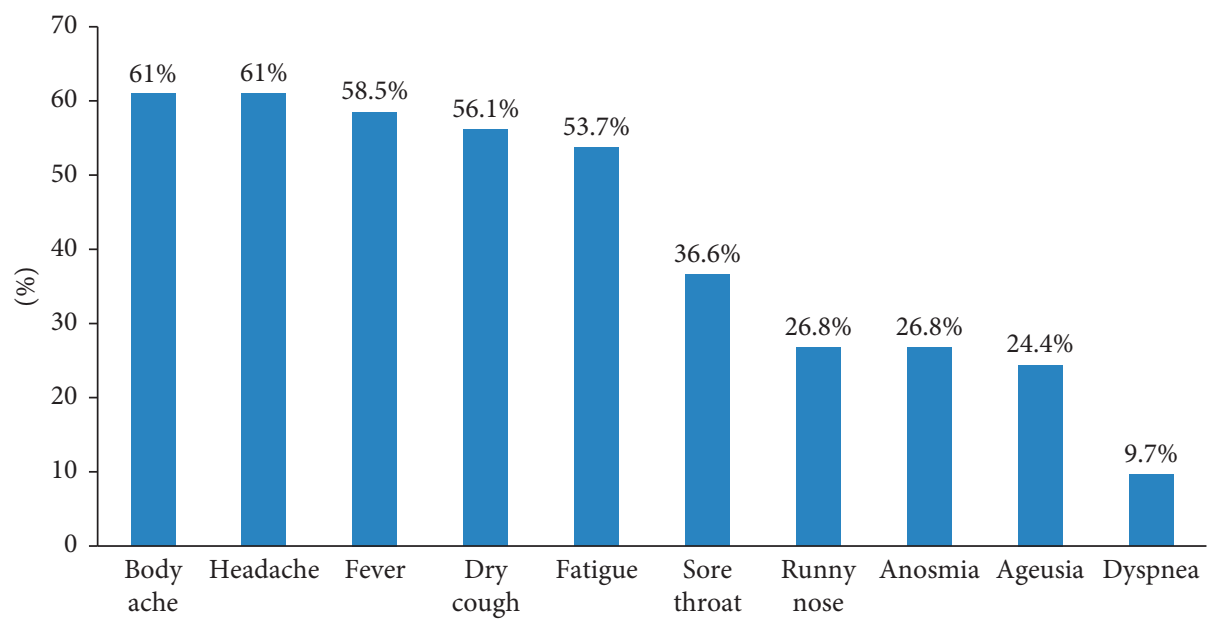

Figure 2: Clinical presentations among HCWs who tested positive for antibody.

Our study demonstrates higher seropositivity among the high-risk group and moderate risk group in comparison to the low-risk group, which is in accordance with the findings of Varona et al. in Spain, in which it is demonstrated that high-risk and moderate-risk groups presented a higher probability of seropositivity as compared to the low-risk group [14].

In the study population, $51.2 \%$ of seropositive HCWs in our study had a history of RT-PCR confirmed SARS-CoV-2 infection, and the remaining $48.8 \%$ never obtained positive RT-PCR results until the time of the investigation. Similarly, in a study performed by Varona et al. in Spain, 67\% of seropositive HCWs had no previous diagnosis of SARSCoV-2 infection before serodiagnosis [14]. Our study showed a significant association between the antibody screening and the history of SARS-CoV-2 infection among HCWs, suggesting that HCWs previously infected with SARS-CoV-2 are more likely to be seropositive, which is in accordance with the study performed in New York, where 93\% of PCR positive HCWs were also seropositive [11]. Similarly, $40 \%$ of seropositive cases in the study performed in Spain did not have a history of SARS-CoV-2 infection, and the same study suggested that those infections might have gone undetected in the diagnostic procedure for SARSCoV-2 infection [29].

65.9\% of seropositive HCWs had flu-like symptoms within the last 6 months. Although the time duration to which the antibodies remain intact following SARS-CoV-2 infection is unclear, the study performed by L'Huillier et al. in Geneva has shown that the antibody titers do not reduce until 6 months after the infection [31]. Studies around the globe, such as a study in Italy [18], a study in Spain [29] and, a study in the UK [19], have shown a significant association between the positive serology and the presence of symptoms. Body ache and headache were the most common symptoms followed by fever and dry cough among the seropositive HCWs in this study. A study performed in a large population of HCWs in the United States has reported the presence of at least one symptom among fever, cough, and dyspnea in a COVID-19 infection [32]. Although body ache, headache, and fever were the most common symptoms among the seropositive, anosmia and ageusia were also reported in $26.8 \%$ and $24.4 \% \mathrm{HCW}$, respectively. Lombardi et al. in Italy found that anosmia, ageusia, and fever were the most common symptoms among SARS-CoV-2-infected HCWs [33]. Also, Rudberg et al. in Sweden and Gracia Basteiro et al. in Spain have discussed ageusia and anosmia as the most predictive symptoms of COVID-19 infection in their studies $[17,29]$. Our study showed a relatively lower percentage of seropositive HCWs showing anosmia and ageusia, which could be described as variation because of subjects' characteristics and geographical variations [18]. We found that $34.1 \%$ of seropositive HCWs were asymptomatic until the time of investigation in the last 6 months. Similarly, Gracia Basteiro et al. reported $23.1 \%$ seropositive $\mathrm{HCW}$ s to be asymptomatic [29]. Asymptomatic infections among HCWs make it difficult to identify the infected ones that could degrade the strategies to control the infection, and therefore, programs for the regular screening of HCWs for COVID-19 are essential in controlling the spread of infection. 
We tested 185 HCWs in our study, among which 41 were seropositive, and all of them had a positive contact history with the COVID-infected patient. The positive contact history was significantly associated with the positive serology, which suggests that the HCWs with positive contact history are more likely to be seropositive. It was in accordance with the findings of Rudberg et al., where the HCWs in contact with COVID-19 patients had higher seroprevalence than the HCWs in contact with non-COVID-19 patients [17]. A study performed by Lombardi et al. in Italy also found that the HCWs with a positive history of being in contact with COVID-19-infected patients are more likely to be seropositive [18]. The significant contribution of this study is that it has demonstrated the infection and seroprevalence of healthcare workers after approximately one year of exposure to the hospital environment by different risk groups of HCWs.

This study has some limitations. This study was a timeframed, single-center study with a relatively small size population. At the time of collection, some of the participants had either been infected recently and had not developed an antibody response or some participants had been infected previously but antibody levels had declined, which could have given an underestimated prevalence rate. Furthermore, it could have been better if we could use ELISA or CLIA for antibody testing.

\section{Conclusion}

The seroprevalence among HCWs was found to be $22.2 \%$. The burden of SARS-CoV-2 infection among HCWs seems to be high, and HCWs are at risk of acquiring infection in the workplace. High prevalence among HCWs was explained by the long exposure of HCWs to the SARS-CoV-2 infected patients. The study revealed that symptomatic infections are more likely to develop strong antibodies.

The relatively higher seroprevalence in our study could be because of the lack of programs for the regular screening of HCWs for SARS-CoV-2 infection, long-term exposure of HCWs with the infected cases (symptomatic or asymptomatic), and the lack of proper PPE. Furthermore, this study recommends that programs for the regular screening of HCWs for SARS-CoV-2 infection regardless of symptoms should be implemented in all the healthcare settings that could help in monitoring the transmission dynamics and the evaluation of infection control policies. Also, this state of the problem could highlight the steps to be taken forward to be prepared for such outbreaks, ensuring that the diagnostic ability and PPE supply is adequate.

\section{Abbreviations}

SARS- $\quad$ Severe acute respiratory syndrome coronavirus

CoV-2: 2

COVID-19: Coronavirus disease 2019

WHO: World Health Organization

HCWs: Healthcare workers

MMTH:
Manmohan Memorial Medical College and

Teaching Hospital

SPSS: $\quad$ Statistical Package for Social Sciences

IQR: Interquartile range

ELISA: $\quad$ Enzyme-linked immuno-sorbent assay

CLIA: Chemiluminescence immunoassay

PPE: $\quad$ Personal protective equipment.

\section{Data Availability}

All data generated during this study are presented in this paper and accessible on reasonable request.

\section{Ethical Approval}

This research work was approved by the Institutional Review Committee of Manmohan Memorial Institute of Health Sciences (MMIHS-IRC 491), Kathmandu, Nepal.

\section{Consent}

Informed written consent was taken from the healthcare workers before participating in the study. Data regarding personal information were coded and kept confidential.

\section{Conflicts of Interest}

The authors declare that they have no conflicts of interest.

\section{Authors' Contributions}

BPR, DDS, and MPB conceived the design of the study, reviewed literature, and performed necessary interventions including laboratory investigations. $\mathrm{SA}, \mathrm{SP}_{1}, \mathrm{MC}$, and $\mathrm{PB}$ participated in data collection and laboratory procedures. SA and $\mathrm{SP}_{2}$ analyzed the data. $\mathrm{SA}, \mathrm{SP}_{2}$, and $\mathrm{BPR}$ prepared the manuscript. All authors contributed to drafting and critically revising the paper and agreed to be accountable for all aspects of the work.

\section{Acknowledgments}

The authors thank all the healthcare workers participating in this study. Our special thanks go to all the laboratory staff, management, and officials of Manmohan Memorial Medical College and Teaching Hospital, Kathmandu, for providing the opportunity to carry out this research work. Serology kits for this research work were provided by the management of Manmohan Memorial Medical College and Teaching Hospital, Kathmandu, Nepal.

\section{References}

[1] World Health Organization, Naming the Coronavirus Disease (COVID-19) and the Virus that Causes it, World Health Organization, Geneva, Switzerland, 2020.

[2] C. Huang, Y. Wang, X. Li et al., "Clinical features of patients infected with 2019 novel coronavirus in Wuhan, China," The lancet, vol. 395, no. 10223, pp. 497-506, 2020.

[3] Y.-R. Guo, Q.-D. Cao, Z.-S. Hong, Y.-Y. Tan, S.-D. Chen, and H.-J. Jin, "The origin, transmission and clinical therapies on 
coronavirus disease 2019 (COVID-19) outbreak-an update on the status," Military Medical Research, vol. 7, no. 1, pp. 1-10, 2020.

[4] World Health Organization, WHO Coronavirus Disease (COVID-19) Dashboard, World Health Organization, Geneva, Switzerland, 2020.

[5] S. Wendel, J. M. Kutner, R. Machado, R. Fontão-Wendel, C. Bub, and R. Fachini, "Screening for SARS-CoV-2 antibodies in convalescent plasma in Brazil: preliminary lessons from a voluntary convalescent donor program," Transfusion, vol. 60, no. 12, pp. 2938-2951, 2020.

[6] Control CfD, Prevention, Interim Guidelines for COVID-19 Antibody Testing, 2020, https://www.cdcgov/coronavirus/ 2019-ncov/lab/resources/antibody-tests-guidelines.html.

[7] P. Kellam and W. Barclay, "The dynamics of humoral immune responses following SARS-CoV-2 infection and the potential for reinfection," Journal of General Virology, vol. 101, no. 8, Article ID jgv001439, 2020.

[8] M. Psichogiou, A. Karabinis, I. D. Pavlopoulou et al., "Antibodies against SARS-CoV-2 among health care workers in a country with low burden of COVID-19," PLoS One, vol. 15, no. 12, Article ID e0243025, 2020.

[9] D. Poulikakos, S. Sinha, and P. A. Kalra, "SARS-CoV-2 antibody screening in healthcare workers in a tertiary centre in North West England," Journal of Clinical Virology, vol. 129, 2020.

[10] D. Steensels, E. Oris, L. Coninx et al., "Hospital-wide SARSCoV-2 antibody screening in 3056 staff in a tertiary center in Belgium," JAMA, vol. 324, no. 2, pp. 195-197, 2020.

[11] J. Moscola, G. Sembajwe, M. Jarrett et al., "Prevalence of SARS-CoV-2 antibodies in health care personnel in the New York city area," JAMA, vol. 324, no. 9, pp. 893-895, 2020.

[12] Z. Al Maskari, A. Al Blushi, F. Khamis et al., "Characteristics of healthcare workers infected with COVID-19: a cross-sectional observational study," International Journal of Infectious Diseases, vol. 102, pp. 32-36, 2021.

[13] E. Lahner, E. Dilaghi, C. Prestigiacomo et al., "Prevalence of sars-cov-2 infection in health workers (HWs) and diagnostic test performance: the experience of a teaching hospital in Central Italy," International Journal of Environmental Research and Public Health, vol. 17, no. 12, p. 4417, 2020.

[14] J. F. Varona, R. Madurga, F. Peñalver et al., "Seroprevalence of SARS-CoV-2 antibodies in over 6000 healthcare workers in Spain," International Journal of Epidemiology, vol. 50, no. 2, pp. 400-409, 2021.

[15] Population GoNMoHa, 2020, Enhanced Surveillance on SeroPrevalence of SARS-COV-2 in General Population.

[16] S. von Huth, S. T. Lillevang, B. T. Røge et al., "SARS-CoV-2 seroprevalence among 7950 healthcare workers in the region of Southern Denmark," International Journal of Infectious Diseases, vol. 112, pp. 96-102, 2021.

[17] A.-S. Rudberg, S. Havervall, A. Månberg, A. J. Falk, K. Aguilera, and H. Ng, "SARS-CoV-2 exposure, symptoms and seroprevalence in health care workers," medRxiv, vol. 2020, 2020.

[18] A. Lombardi, D. Mangioni, D. Consonni et al., "Seroprevalence of anti-SARS-CoV-2 IgG among healthcare workers of a large university hospital in Milan, Lombardy, Italy: a crosssectional study," BMJ Open, vol. 11, no. 2, Article ID e047216, 2021.

[19] A. M. Shields, S. E. Faustini, M. Perez-Toledo, S. Jossi, E. Aldera, and J. D. Allen, "SARS-CoV-2 seroconversion in health care workers," medRxiv, vol. 2020, 2020.
[20] P. Galanis, I. Vraka, D. Fragkou, A. Bilali, and D. Kaitelidou, "Seroprevalence of SARS-CoV-2 antibodies and associated factors in healthcare workers: a systematic review and metaanalysis," Journal of Hospital Infection, vol. 108, pp. 120-134, 2021.

[21] A. Hossain, S. M. Nasrullah, Z. Tasnim, M. K. Hasan, and M. M. Hasan, "Seroprevalence of SARS-CoV-2 IgG antibodies among health care workers prior to vaccine administration in Europe, the USA and East Asia: a systematic review and meta-analysis," EClinicalMedicine, vol. 33, Article ID 100770, 2021.

[22] C. F. Houlihan, N. Vora, T. Byrne, D. Lewer, J. Heaney, and D. A. Moore, "SARS-CoV-2 virus and antibodies in front-line Health Care Workers in an acute hospital in London: preliminary results from a longitudinal study," medRxiv, vol. 2020, 2020.

[23] N. M. Vaselli, D. Hungerford, B. Shenton, A. Khashkhusha, N. A. Cunliffe, and N. French, "The seroprevalence of SARS-CoV-2 during the first wave in Europe 2020: a systematic review," PloS one, vol. 16, no. 11, Article ID e0250541, 2021.

[24] S. A. Müller, R. R. Wood, J. Hanefeld, and C. El-Bcheraoui, "Seroprevalence and risk factors of COVID-19 in healthcare workers from 11 African countries: a scoping review and appraisal of existing evidence," Health Policy and Planning, 2021.

[25] O. Olayanju, O. Bamidele, F. Edem et al., "SARS-CoV-2 seropositivity in asymptomatic frontline health workers in Ibadan, Nigeria," The American Journal of Tropical Medicine and Hygiene, vol. 104, no. 1, pp. 91-94, 2021.

[26] A. M. Kammon, A. A. El-Arabi, E. A. Erhouma, T. M. Mehemed, and O. A. Mohamed, "Seroprevalence of antibodies against SARS-CoV-2 among public community and health-care workers in Alzintan City of Libya," medRxiv, 2020.

[27] A. Mostafa, S. Kandil, M. H. El-Sayed et al., "Universal COVID-19 screening of 4040 health care workers in a resource-limited setting: an Egyptian pilot model in a university with 12 public hospitals and medical centers," International Journal of Epidemiology, vol. 50, no. 1, pp. 50-61, 2021.

[28] W. A. Halatoko, Y. R. Konu, F. A. Gbeasor-Komlanvi et al., "Prevalence of SARS-CoV-2 among high-risk populations in Lomé (Togo) in 2020," PLoS One, vol. 15, no. 11, Article ID e0242124, 2020.

[29] A. L. Garcia-Basteiro, G. Moncunill, M. Tortajada, M. Vidal, C. Guinovart, and A. Jiménez, "Seroprevalence of antibodies against SARS-CoV-2 among health care workers in a large Spanish reference hospital," medRxiv, vol. 2020, 2020.

[30] J. T. Wilkins, E. L. Gray, A. Wallia et al., "Seroprevalence and correlates of SARS-CoV-2 antibodies in health care workers in Chicago," Open Forum Infectious Diseases, vol. 8, no. $1,2021$.

[31] A. G. L'Huillier, B. Meyer, D. O. Andrey, I. Arm-Vernez, S. Baggio, and A. Didierlaurent, "Antibody persistence in the first 6 months following SARS-CoV-2 infection among hospital workers: a prospective longitudinal study," Clinical Microbiology and Infection, vol. 27, no. 5, 2021.

[32] S. L. Burrer, M. A. de Perio, M. M. Hughes et al., "Characteristics of health care personnel with COVID-19-United States, february 12-april 9, 2020," MMWR Morbidity and Mortality Weekly Report, vol. 69, no. 15, pp. 477-481, 2020.

[33] A. Lombardi, D. Consonni, M. Carugno et al., "Characteristics of 1573 healthcare workers who underwent nasopharyngeal 
swab testing for SARS-CoV-2 in Milan, Lombardy, Italy," Clinical Microbiology and Infection: The Official Publication of the European Society of Clinical Microbiology and Infectious Diseases, vol. 26, no. 10, p. 1413, 2020. 